\title{
Synthesis, mechanical, thermal and chemical properties of polyurethanes based on cardanol
}

\author{
C V MYTHILI, A MALAR RETNA and S GOPALAKRISHNAN* \\ Department of Chemistry, Manonmaniam Sundaranar University, Abishekapatti, Tirunelveli 627 012, India
}

MS received 28 August 2003; revised 19 February 2004

\begin{abstract}
Cardanol, an excellent monomer for polymer production, has been isolated from CNSL and allowed to react with formaldehyde in a particular mole ratio in the presence of glutaric acid catalyst to give high-ortho novolac resin. Such characterized polyol has been condensed with diphenylmethane diisocyanate to produce rigid polyurethane. A commercially available polyol, polypropylene glycol-2000 (PPG-2000), has also been condensed with diphenylmethane diisocyanate and polyol to produce tough polyurethane. These polyurethanes were characterized with respect to their resistance to chemical reagents and mechanical properties such as tensile strength, percentage elongation, tear strength and hardness. Differential thermal analysis (DTA) and thermo-gravimetric analysis (TGA) were undertaken for thermal characterization.
\end{abstract}

Keywords. Cardanol; polyurethane; diphenylmethane diisocyanate; polypropylene glycol-2000.

\section{Introduction}

In the last 20 years, considerable attention has been paid to the synthesis of polymers based on cardanol (Patel et al 1989; Pillot et al 1989; Manjula et al 1991, 1992), a natural meta-substituted phenol from cashew nut shell liquid. There have been a large number of publications on modification of cardanol using the reactivity of hydroxy phenyl group (Ghatge and Vailva 1975; Ghatge and Shinde 1979; Balakrishna et al 1990; Manjula et al 1990; John and Pillai 1992; Madhusudhan and Murthy 1992; Antony 1993). However, reports concerning the cardanol formaldehyde resins leading to novel polyurethanes are very limited. In the present study, cardanol formaldehyde resin has been prepared and it is made to react with diphenylmethane diisocyanate to form rigid polyurethane. Tough polyurethane has also been prepared using the commercially available polypropylene glycol-2000 (PPG-2000) along with the cardanol formaldehyde resin and the diisocyanate in a particular mole ratio.

\section{Experimental}

\subsection{Materials and methods}

2.1a Materials: Cardanol was obtained from M/s Rishabh Resins \& Chemicals Ltd., Hyderabad. Formaldehyde (40\% solution) and methanol were received from $\mathrm{M} / \mathrm{s} \mathrm{BDH}$ (India) Ltd., glutaric acid and diphenylmethane diisocyanate were received from E. Merck (Germany), PPG-2000

\footnotetext{
*Author for correspondence
}

was received from Aldrich Chemicals (USA), and dibutyl tindilaurate was received from Fluka Chemie (Germany). The chemicals were used as received.

2.1b Methods: Ultraviolet spectral analysis was carried out in a Shimadzu UV 2100 UV-visible spectrophotometer. Infrared spectra were taken in a JASCO FT-IR spectrophotometer by $\mathrm{KBr}$ pellet method. ${ }^{1} \mathrm{H}-\mathrm{NMR}$ spectra was recorded using an R 248.H.Hitachi $300 \mathrm{MHz}$ NMR spectrophotometer. Thin layer chromatographic separation was carried out in three different solvent systems. Specific gravity, iodine value, hydroxyl value and intrinsic viscosity of the resin were determined according to the IS standard 840-1964. Molecular weight of the resin was determined by gel permeation chromatography using $\mu$-styragel columns, $100 \AA$ and $500 \AA$, UV detector and $280 \mathrm{~nm}$ filter. The tear strength of the polyurethanes was determined using a Zwick Universal testing machine (UTM) as per ASTM standard D624. Indentation hardness (shore A) was determined as per ASTM standard D2240. Hardness tester durometer was used. Tensile strength of the polyurethanes was determined as per ASTM standard D412. The polyurethanes were subjected to differential thermal analysis (DTA)/thermogravimetric analysis (TGA) studies at a rate of $10 \mathrm{~K} / \mathrm{min}$ in air/nitrogen using Rheometric scientific STA1500+ machine. Acid, alkali and solvent resistance were estimated according to ASTM standard D3137, C267. Polyurethane samples $(3 \times 1 \times 0 \cdot 1 \mathrm{~cm})$ were immersed in the medium $(100 \mathrm{ml})$ for a total duration of 60 days under ambient conditions. The medium was changed and fresh medium was added at an interval of one week. The loss of weight was determined using an electronic weighing balance. 


\subsection{Synthesis of cardanol formaldehyde resin}

Monoene constituent of cardanol had been separated by argentation liquid chromatography method (Sood et al 1986). $100.7 \mathrm{~g}$ of cardanol, $22.5 \mathrm{ml}$ of formaldehyde (40\% solution), and $1 \%$ glutaric acid catalyst in methanol were refluxed at $120 \pm 5^{\circ} \mathrm{C}$ for $3 \mathrm{~h}$ and then at $150 \pm 5^{\circ} \mathrm{C}$ for $2 \mathrm{~h}$. The initial $\mathrm{pH}$ of the reaction mixture was 4 which was lowered to 2 after the completion of condensation. The resin was purified by dissolving in toluene and by precipitating it with distilled water. Major fraction was collected and dried using a rotary evaporator under vacuum and analysed.

\subsection{Synthesis of rigid polyurethane (CRPU)}

Rigid polyurethane was synthesized using freshly prepared cardanol formaldehyde resin. The vacuum dried $0 \cdot 1$ mole of cardanol formaldehyde resin and 0.35 mole of diphenylmethane diisocyanate (MDI) were reacted, keeping the isocyanate index ( $\mathrm{NCO} / \mathrm{OH}$ molar ratio) constant at 1.4. Hydroxyl number of the resin was determined using the equation

$$
56 N=(w / W) \times(\text { molecular weight }+42 N),
$$

where $\mathrm{N}$ is the number of hydroxyl groups, $w$ the amount of $\mathrm{KOH}$ required for the hydrolysis of acetyl derivative of the resin and $W$ the weight of acetyl derivative of the resin. The reaction was carried out at room temperature in the presence of the catalyst, dibutyl tindilaurate $(0 \cdot 12 \mathrm{wt} \%)$. The polymer was then allowed to cure for $24 \mathrm{~h}$ in a flat surface without any disturbance. The polymer was again cured in a vacuum oven at $80^{\circ} \mathrm{C}$ for $48 \mathrm{~h}$ and used to evaluate its properties.

\subsection{Synthesis of tough polyurethane (CTPU)}

The vacuum-dried $0 \cdot 1$ mole of cardanol formaldehyde resin, 0.49 mole of diphenylmethane diisocyanate (MDI), and 0.1 mole of commercially available polypropylene glycol, PPG-2000 were reacted, keeping the isocyanate index $(\mathrm{NCO} / \mathrm{OH}$ molar ratio) constant at 1.4. The reaction was carried out as indicated in the case of rigid polyurethane and the tough polyurethane was cured.

\section{Results and discussion}

It has been reported (Sathyalekshmi and Gopalakrishnan 2000) that in the case of resin having higher mole ratio (1: $0 \cdot 9)$, the degree of condensation between methylolated cardanol in presence of adipic acid catalyst to form multi nuclear resin is high and the product is ortho-para substituted. In the present study, the formylation and condensation of cardanol with formaldehyde in the mole ratio
(1: 0.9$)$ in the presence of glutaric acid catalyst also leads to ortho-para substituted product.

\section{$3.1 U V$ and IR spectroscopy of cardanol formaldehyde resin}

Cardanol shows sharp peak at $285 \mathrm{~nm}$ which is attributed to aromatic phenyl ring. Substitution at the phenyl ring by condensation increases the wavelength to $292 \mathrm{~nm}$, which is due to bathochromic effect (Knowles 1984; Denny and Sinclair 1991). IR spectral data of cardanol formaldehyde resin reveal not only the condensation of methylolated cardanol, but also the degree of ortho and para substitution (Sathyalekshmi and Gopalakrishnan 2000). The peak at $967 \mathrm{~cm}^{-1}$ is due to substitution in benzene nuclei and the peaks at $909 \mathrm{~cm}^{-1}$ and $697 \mathrm{~cm}^{-1}$ are due to three adjacent hydrogen atoms in the benzene nuclei. The sharp peak at $767 \mathrm{~cm}^{-1}$ indicates the ortho substitution at benzene nuclei. The sharp peak at $863 \mathrm{~cm}^{-1}$ indicates the para substitution at benzene nuclei (see figure 1).

\section{$3.2{ }^{1} H-N M R$ spectroscopy of cardanol formaldehyde resin}

In the ${ }^{1} \mathrm{H}-\mathrm{NMR}$ spectra of cardanol formaldehyde resin, the peak at $6 \cdot 60-7 \cdot 20 \delta$ is due to aryl protons of benzene nuclei, the peak around the region $6.45 \delta$ is due to the phenolic hydroxyl, the peak at $4 \cdot 90-5 \cdot 30 \delta$ is due to methylene $\left(\mathrm{C}=\mathrm{CH}_{2}\right)$ proton of long alkyl side chain originally present in cardanol and the peak at $0 \cdot 88-2 \cdot 70 \delta$ is due to the long aliphatic side chain. The small peak at $0.80 \delta$ is due to terminal methyl group of the chain. The strong peak at $1.30 \delta$ is attributed to the long chain (more than five methylene groups) of the side chain. The peak at $3.6 \delta$ also indicates methylene proton of $\mathrm{C}_{6} \mathrm{H}_{5}-\mathrm{CH}_{2}-$ $\mathrm{C}_{6} \mathrm{H}_{5}$ for the bridge between the phenyl rings. All these spectral data indicate that the condensation of methylolated cardanol has been completed under experimental condition (see figure 2).

\subsection{Thin layer chromatographic analysis of cardanol formaldehyde resin}

Thin layer chromatographic study of the resin in three different solvent systems showed a distinct single spot, indicating the purity of the sample. $R_{\mathrm{f}}$ value for cardanol formaldehyde resin is different from that of cardanol indicating the formation of the condensation product, multi nuclear novolac resin (see table 1).

\subsection{Physico-chemical properties of cardanol formaldehyde resin}

Physico-chemical properties are presented in table 1. The cardanol formaldehyde resin possesses higher specific 
gravity and viscosity due to higher degree of condensation between cardanol and formaldehyde. The low iodine value in comparison with that of cardanol may be due to steric hindrance of adjacent bulky groups to the olefinic addition of iodine monochloride during the estimation of iodine value. The number of hydroxyl groups present in the resin are only phenolic $-\mathrm{OH}$ groups. The IR spectral analysis, hydroxyl number and molecular weight determination enables to evolve the molecular structure of cardanol formaldehyde resin (see figure 3 ).

\subsection{Mechanism for the synthesis of polyurethanes}

The condensation reaction between cardanol formaldehyde resin and di-isocyanate can lead to the formation of stable urethane linkages. The condensation reaction was found to be exothermic. Dibutyl tindilaurate catalyst was used and the reaction was completed within $10 \mathrm{~min}$. The ratio of concentrations of isocyanate to hydroxy groups $(1: 1.4)$ was chosen so that the excess of isocyanate present leads to the formation of terminal isocyanate groups as suggested by the following reaction:

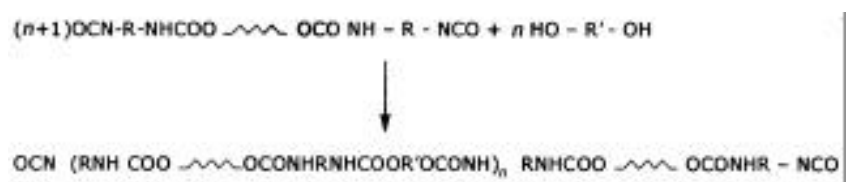

The final curing of the reaction product leads to the formation of allophanate linkages with the reaction in- volving terminal isocyanate groups with active hydrogen present in urethane groups of the polymer as follows:

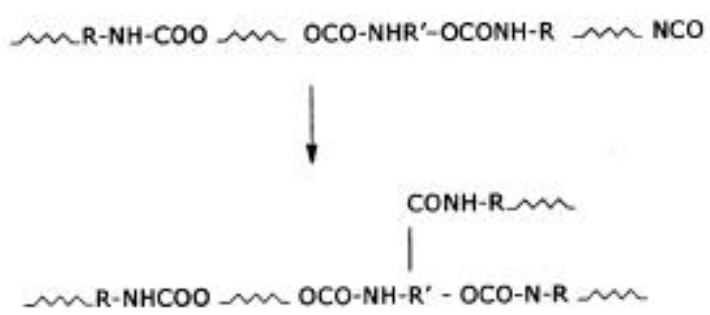

$$
\begin{aligned}
& R \cdot \stackrel{P}{P}-\stackrel{+}{+}=\ddot{O}+M^{2+} \rightleftharpoons R-\underset{M^{2+}}{=}=C=0 \\
& \text { (1) }
\end{aligned}
$$

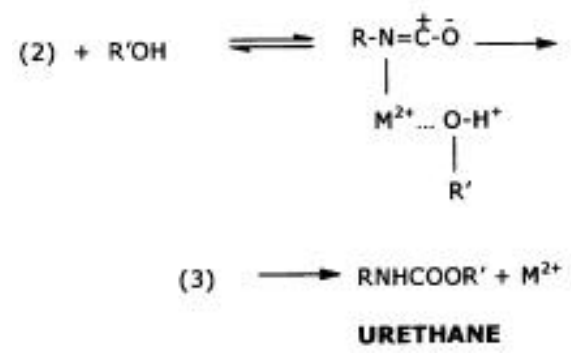

\subsection{Spectral properties of polyurethanes}

IR spectrum of CRPU and CTPU showed characteristic absorption at $3414 \mathrm{~cm}^{-1}$ corresponding to urethane link-

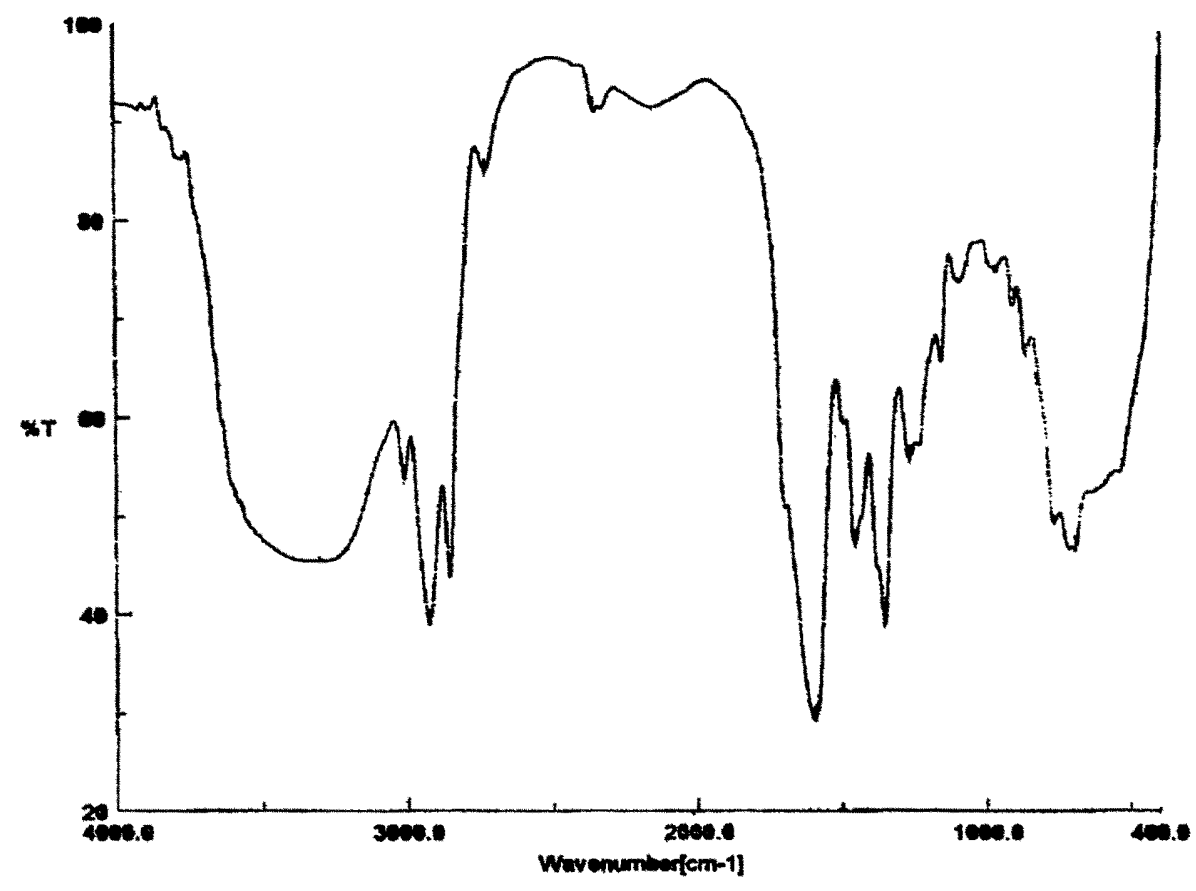

Figure 1. IR spectrum of cardanol formaldehyde resin. 


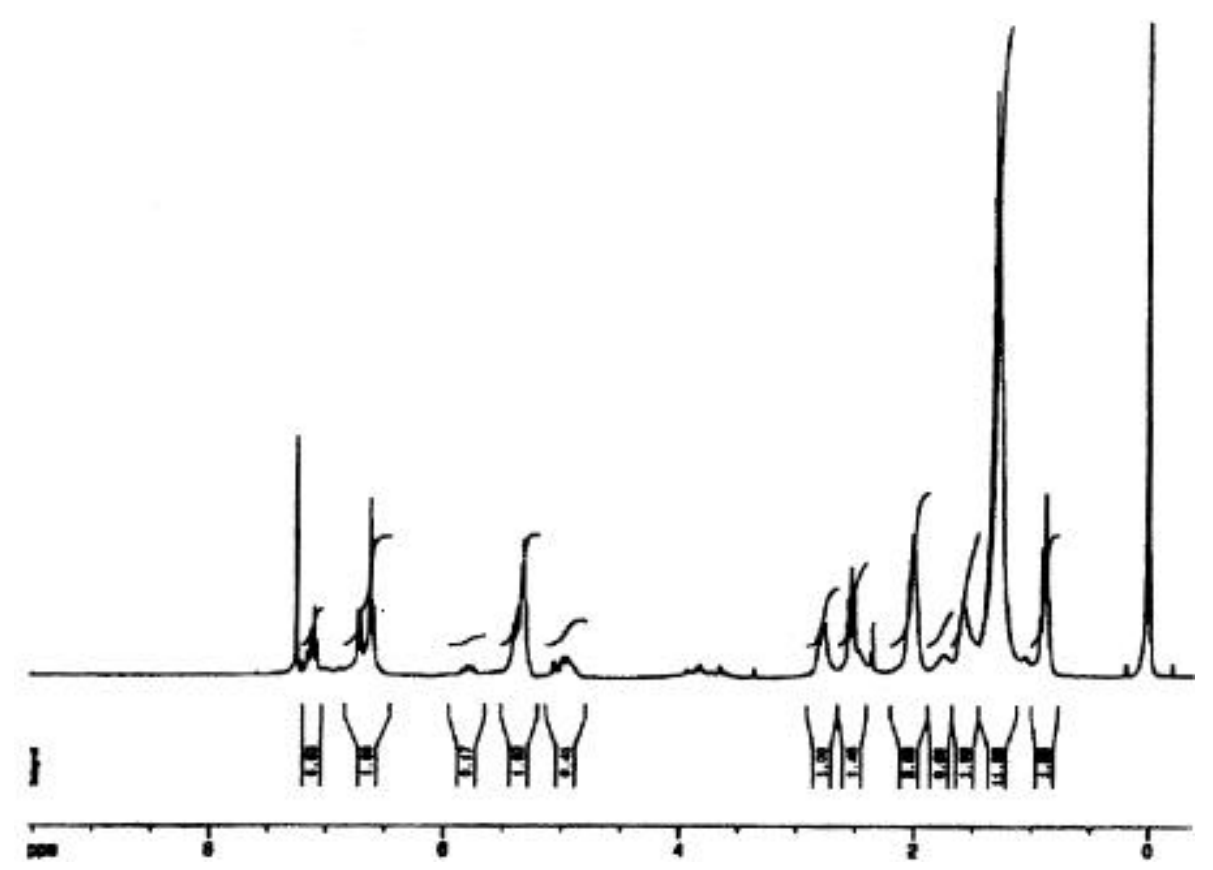

Figure 2. $\quad{ }^{1} \mathrm{H}-\mathrm{NMR}$ spectrum of cardanol formaldehyde resin.

Table 1. Physico-chemical properties of cardanol and cardanol formaldehyde resin.

\begin{tabular}{lll}
\hline Properties & Cardanol & Cardanol formaldehyde resin \\
\hline Colour & Dark brown & Dark brown \\
Odour & Phenolic & Phenolic \\
Specific gravity $\left(\mathrm{g} / \mathrm{cc}\right.$ at $\left.30^{\circ} \mathrm{C}\right)$ & $0 \cdot 9285$ & 0.9333 \\
Viscosity (intrinsic) & $3 \cdot 13 \times 10^{-2}$ & $3.28 \times 10^{-2}$ \\
Hydroxyl value & $181 \cdot 5$ & $157 \cdot 2$ \\
Iodine value & $223 \cdot 2$ & $221 \cdot 8$ \\
Molecular weight & 302 & 1550 \\
Number of hydroxyl groups & 1 & 5 \\
Thin layer chromatography: $R_{\mathrm{f}}$ values & & \\
(a) Petroleum ether : diethyl ether $(7: 3)$ & $0 \cdot 82$ & $0 \cdot 78$ \\
(b) 100\% benzene & $0 \cdot 77$ & 0.75 \\
(c) Benzene : chloroform $(1: 1)$ & 0.81 & $0 \cdot 76$ \\
\hline
\end{tabular}

age ( $-\mathrm{NH}$ stretching), $1743 \mathrm{~cm}^{-1}$ corresponding to $\mathrm{C}=\mathrm{O}$ stretching (free) in urethane, $1605 \mathrm{~cm}^{-1}$ corresponding to $\mathrm{C}=\mathrm{O}$ stretching (bonded) in urethane and $1543 \mathrm{~cm}^{-1}$ corresponding to $\mathrm{N}-\mathrm{H}$ bending in urethane (see figure 4 ).

\subsection{Mechanical properties of polyurethanes}

Mechanical properties such as elongation at break (\%), tensile strength, tear strength, shore hardness are furnished in table 2. Tensile strength and tear strength are largely influenced by the presence of aromatic groups, long alkyl chain, branching and cross linking and also degree of secondary bonding forces. The tear test data of the rigid polyurethane (CRPU) shows that this polyurethane crumbles during tear test. The addition of commercial polyol, (PPG-2000) along with cardanol formaldehyde

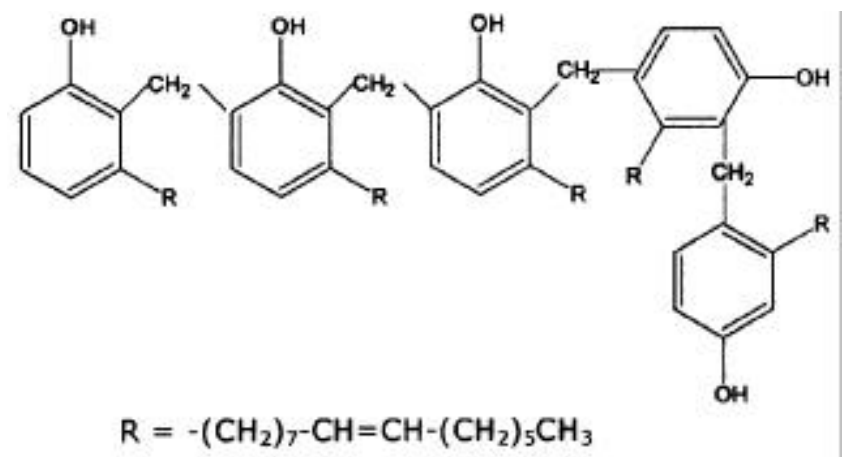

Figure 3. Molecular structure of cardanol formaldehyde resin.

resin introduces some degree of flexibility in the tough polyurethane (CTPU). This is attributed to lower molecular weight between the cross links and higher cross link 


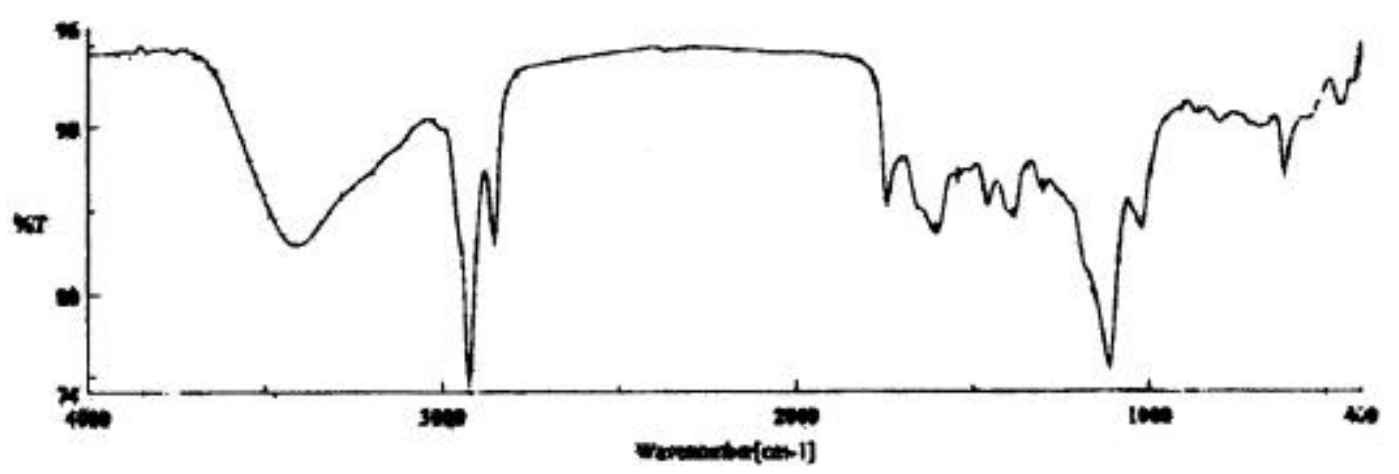

Figure 4. IR spectrum of CRPU.

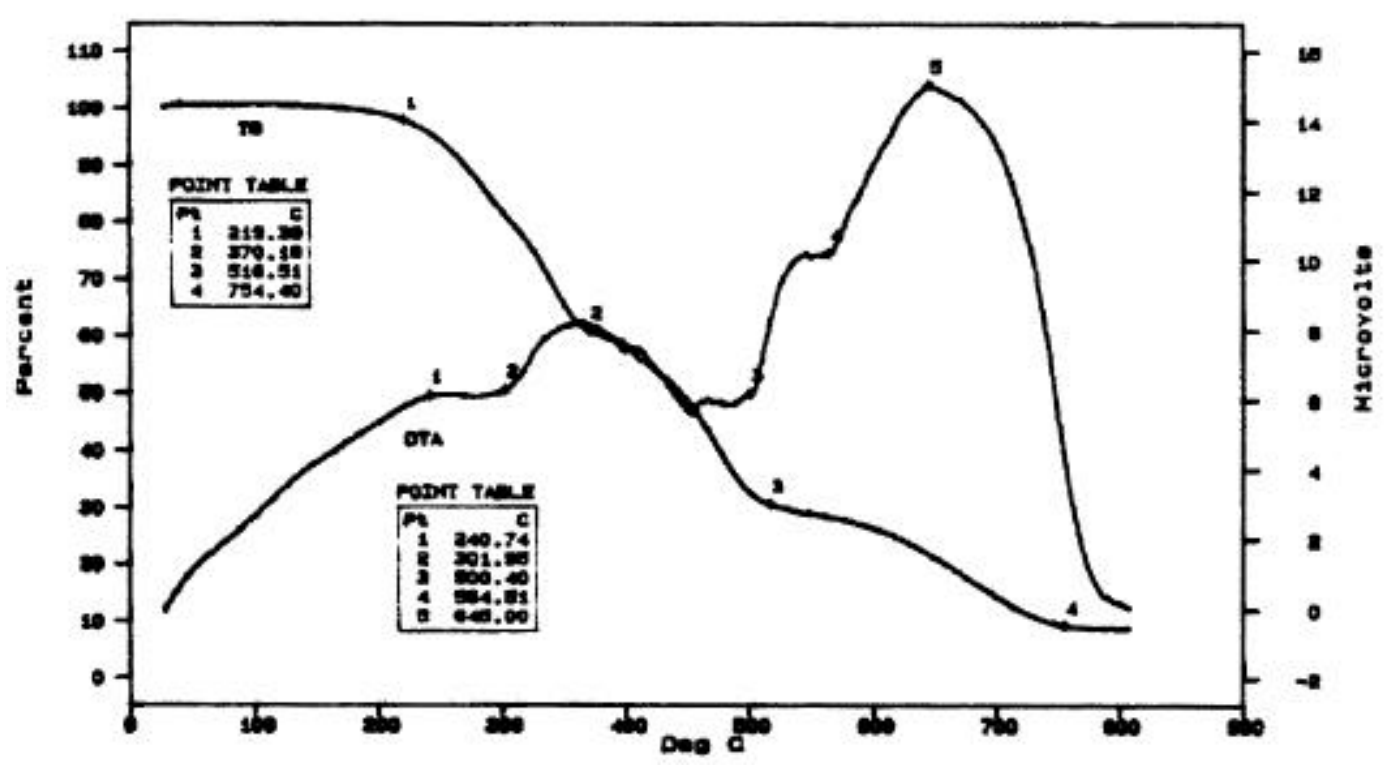

Figure 5. DTA/TGA of CRPU.

density of the rigid polyurethane. In the present work, the rigid polyurethane possesses higher shore hardness when compared to that of the tough polyurethane. It has been reported that hardness is primarily governed by the hard segment concentration (Petravic et al 1991). Lower tensile strength in rigid polyurethanes is due to low molecular weight between the cross links and higher cross link density. Increase in elongation in tough polyurethane is due to the flexibility of the chain introduced by the polyol.

\subsection{Thermal properties of polyurethanes}

Thermal properties of the present polyurethanes are influenced mainly by molecular weight between the cross links, degree of segments in stiff sequence, segments in flexible sequence and elastically active branch points as has been advocated by previous researchers for polyurethanes (Dusek et al 1990). The segments which constitute stiff sequence are biuret and allophanate. Hard segments
Table 2. Mechanical properties of rigid and tough polyurethanes.

\begin{tabular}{lcccc}
\hline & $\begin{array}{c}\text { Tensile } \\
\text { strength } \\
(\mathrm{MPa})\end{array}$ & $\begin{array}{c}\text { Tear } \\
\text { strength } \\
(\mathrm{kN} / \mathrm{m})\end{array}$ & $\begin{array}{c}\text { \% Elongation } \\
\text { at break }\end{array}$ & $\begin{array}{c}\text { Hardness } \\
\text { shore (A) }\end{array}$ \\
\hline Colyurethane & Brittle & Brittle & Brittle & 88 \\
CTPU & 21.47 & 120 & 75 & 65 \\
\hline
\end{tabular}

existing between urethane linkages comprising cardanol formaldehyde resin and diisocyanate, i.e. the condensation product of resin and diisocyanate, also imparts stiffness in the chain. The flexible soft segment is the polyol segment which starts either from urethane or allophanate linkages. The elastically active branch points comprise biuret and allophanate (Dusek et al 1990). In the present study, DTA thermograms of cured rigid and tough polyurethanes are presented in figures 5 and 6 , respectively. DTA thermogram does not show any endothermic peak 


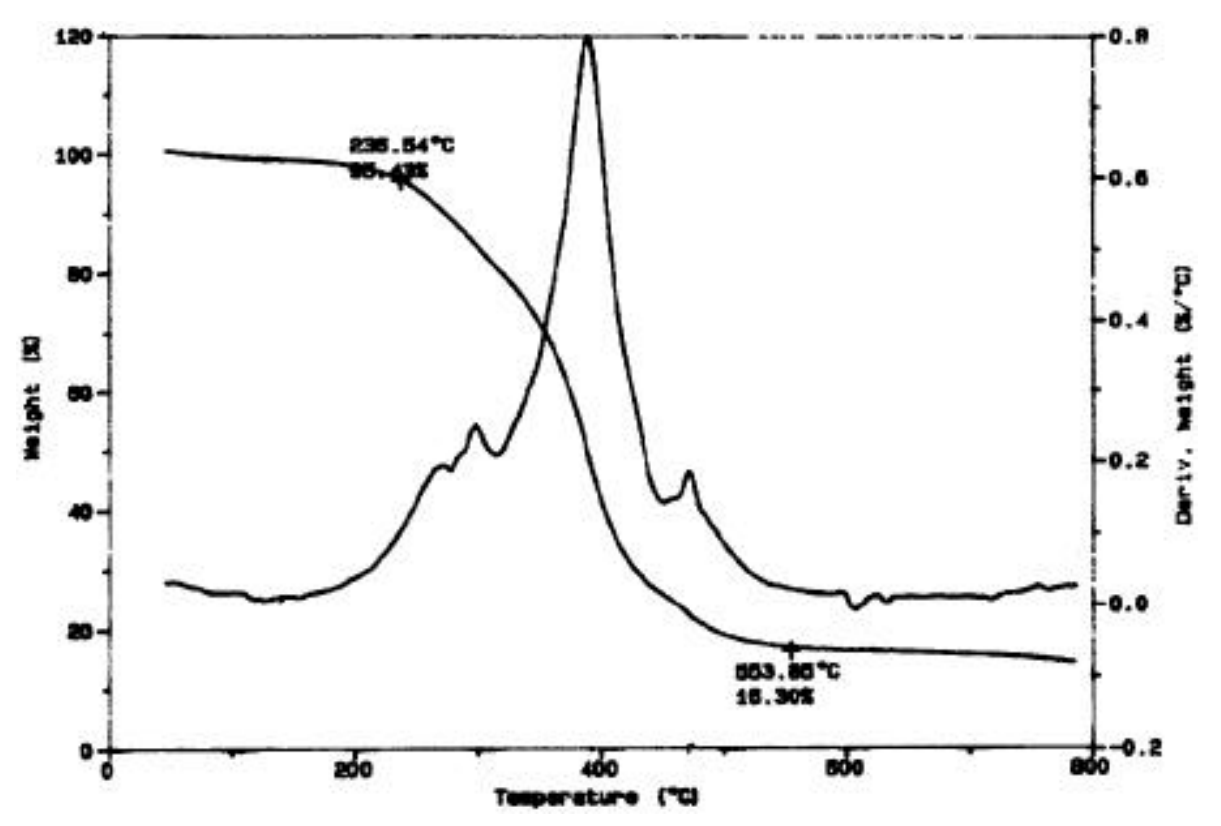

Figure 6. DTA/TGA of CTPU.

Table 3. Chemical resistance of rigid and tough polyurethanes.

\begin{tabular}{lcc}
\hline & \multicolumn{2}{c}{$\%$ weight loss } \\
\cline { 2 - 3 } Chemical reagent & CRPU & CTPU \\
\hline $1 \mathrm{~N} \mathrm{HCl}$ & 0 & 0.46 \\
$1 \mathrm{~N} \mathrm{NaOH}$ & 0 & 2.00 \\
$1 \mathrm{~N} \mathrm{NaCl}$ & 0 & 0.00 \\
Diethyl ether & 0 & 0.00 \\
Toluene & 0 & 1.64 \\
Ethanol & 0 & 0.00 \\
\hline
\end{tabular}

for softening. But, two exotherms are invariably seen in both the urethanes. The first exotherm for rigid polyurethane at $364.7^{\circ} \mathrm{C}$ and for tough polyurethane at $300^{\circ} \mathrm{C}$ are relatively weak, the second exotherm for rigid polyurethane at $753^{\circ} \mathrm{C}$ and for tough polyurethane at $392.6^{\circ} \mathrm{C}$ are strong. The first exotherm is attributed to the cleavage of long alkyl side chain of phenyl ring and also to the cleavage of allophanate linkages. In cardanol formaldehyde resins, the long alkyl side chain undergoes first cleavage at low temperature as has been reported earlier (Sathyalekshmi 1994). The rigid polyurethane possesses higher degradation temperature in comparison with that of the tough polyurethane, due to higher cross link density. The TGA curves for decomposition of polyurethanes show two-stage decomposition. The TGA analysis of polyurethanes shows that the rigid polyurethane possesses higher thermal stability. 5\% weight loss for rigid polyurethane occurs relatively at higher temperature, $247^{\circ} \mathrm{C}$, when compared to tough polyurethane which occurs at $236 \cdot 5^{\circ} \mathrm{C}$.

\subsection{Chemical resistance of polyurethanes}

The percentage weight loss of both rigid and tough polyurethanes were determined in $1 \mathrm{~N}$ hydrochloric acid, $1 \mathrm{~N}$ sodium hydroxide, diethyl ether, ethanol, toluene, $1 \mathrm{~N}$ sodium chloride etc and the results are furnished in table 3. Both the polyurethanes are stable in acid, alkali, $1 \mathrm{~N}$ sodium chloride, toluene, ether, and ethanol.

\section{Conclusions}

The rigid polyurethane is found to possess higher hardness when compared to the tough polyurethane. Also rigid polyurethane is more stable in chemical reagents when compared to tough polyurethanes and possess good thermal stability.

\section{Acknowledgements}

One of the authors (CVM) thanks the University Grants Commission, New Delhi, the Director of Collegiate Education, Chennai and the Principal, Rani Anna Government College for Women, Tirunelveli, for selecting under FIP programme.

\section{References}

Antony R 1993 J. Polym. Sci. 313187

Balakrishna R S, Satyanarayana M N, Vishwanath B B and Shrisalkar M M 1990 J. Appl. Polym. Sci. 411365 
Denny R C and Sinclair R 1991 Visible and ultraviolet spectroscopy (ed.) D Mowthorpe (Singapore: John Wiley) p. 133

Dusek K, Spirkova M and Havlicek I 1990 Macromolecules 23 1774

Ghatge N D and Shinde B M 1979 J. Elastomers 11148

Ghatge N D and Vailva S V 1975 Die Aggewandte Macromoleculare chemie. $\mathbf{4 3} 1$

John G and Pillai C K S 1992 Makromol. Chem. Rapid. Commun. 13255

Knowles A 1984 Practical absorption spectrometry (eds) A Knowles and C Burgess (London: Chapman and Hall) p. 10

Madhusudhan V and Murthy B G K 1992 Prog. Org. Coat. 20 63

Manjula S, Pillai C K S and Kumar V G 1990 Thermochim. Acta 159255
Manjula S, Pavithran C, Pillai C K S and Kumar V G 1991 J. Mater. Sci. 264001

Manjula S, Kumar V G and Pillai C K S 1992 J. Appl. Polym. Sci. 45309

Patel M S, Patel V S and Patel R G 1989 J. Therm. Anal. 3547

Petravic J S, Havsky M, Dusek K, Vidakavlic M, Jarni J and Banjammin B 1991 J. Appl. Polym. Sci. 1239991

Pillot J P, Vanthanh M D M, Gerval J and Dunogues J 1989 Euro. Polym. J. 25285

Sathyalekshmi K 1994 Polym. Sci. Recent advances (ed.) I S Bhardwajj (New Delhi: Interscience) vol. 2, p. 862

Sathyalekshmi K and Gopalakrishnan S 2000 Plastics, Rubber and Composites 2963

Sood S K, Tyman J H P, Durrani A and Johnson R A 1986 Lipids 21241 